\title{
Synchronous double primary cancer of the penisand urinary bladder
}

\begin{abstract}
Multiple primary cancers (MPC) are a term used to define two distinct cancers arising from two distinct organs, in a single individual. It was Billroth who in 1889 reported the first case of multiple organ cancer sand later Warren and Gates in 1932 defined the criteria for MPC's as

1. Each cancer must be definitively malignant by histopathology,

2. They must be histologically different and

3. The possibility of metastasis among the cancers must be excluded.

Conjecture regarding the definitions of synchronous or simultaneous and metachronous cancers has been resolved, the former means two cancers discovered within 6months of each other while the later stands for two cancers developing in an interval greater than 6 months in a single individual We, here report a case of synchronous bladder and penile cancer in a $75 \mathrm{yr}$ old male. To the best of our knowledge this is only the second report of this combination of MPC, the first by Yong Soo Cho et al in 2010.
\end{abstract}

Volume 4 Issue 5 - 2017

\author{
Harsh Deora,' Govind Sharma, ${ }^{2}$ Vinay Tomar ${ }^{2}$ \\ 'Neurosurgery Resident at Department of Neurosurgery \\ NIMHANS, India \\ ${ }^{2}$ Associate Professor at Department of Urology, Sawai Man \\ Singh Medical College, India
}

\begin{abstract}
Correspondence: Harsh Deora, Neurosurgery Resident at Department of Neurosurgery NIMHANS, Hosur Road, Bangalore-560029, India, Email demo560I@gmail.com
\end{abstract}

Received: October 29, 2016 | Published: July 27, 2017

\section{Introduction}

Multiple primary cancers (MPC) is a term used to define two distinct cancers arising from two distinct organs, in a single individual. It was Billroth ${ }^{1}$ who in 1889 reported the first case of multiple organ cancers and later Warren and Gates ${ }^{2}$ in 1932 defined the criteria for MPC's. There has previously been conjecture regarding the definitions of synchronous or simultaneous and metachronous or successive cancers but, subsequently it has been accepted that the former means two cancers discovered within 6months of each other while the later stands for two cancers developing in an interval greater than 6 months in a single individual. ${ }^{3}$ The point to be noted here being that the definitions are based on time of discovery rather than time of onset of cancers. The incidence of MPC's has been reported to be $3-7 \%{ }^{4}$ Ray et al. ${ }^{5}$ reported that $13.7 \%$ of patients with MPC have genito urinary cancer. Cancer of the bladder is estimated to be the ninth most common cause of cancer worldwide (357 000 cases in $2002)$ and the $13^{\text {th }}$ most numerous cause of death from cancer $(145000$ deaths). ${ }^{6}$ However, penile cancer incidence has been less than $1 \%$ of all male cancers ${ }^{7}$ and thus is extremely rare to occur as a part of MPC.

\section{Case presentation}

A 75year old male, farmer and a heavy smoker (40 to 50 cigarettes in a day from the age of 20yrs) was referred to our institute with the complaint of gross total painless intermittent hematuria for one month. On examination pre-puce was not retractable and though meatus could be seen through pre-pucial opening. Palpation revealed that there was a growth over the glans, which the patient was unaware of (Figure 1). Concomitantly, multiple inguinal lymphadenopathy was present, the largest node being $2 \mathrm{~cm}$ in size on right side. Skin over the nodes was normal. The patient had suffered lower urinary tract symptoms over the preceding 1 year and seemed cachectic, but had not previously reported weight loss or any other specific complaints. He did not have a family history of malignancy, or any other history of medical of surgical treatment in the past. USG of the abdomen revealed a growth found on postero-lateral wall of bladder. CECT (Contrast enhanced computed tomography) abdomen and pelvis advised to patient. On CECT a $4 \times 3 \mathrm{~cm}$ mass was present over right Postero-lateral wall (Figure 2). After completing the preoperative workup the patient was planned for penile growth biopsy and cystoscopic biopsy of bladder tumour. Dorsal slitting was done before cystoscopy and biopsy taken from bladder mass. After that biopsy was taken from the penile growth. Fine needle aspiration of the inguinal lymph nodes was also done. Bladder biopsy revealed a Low grade Transitional cell carcinoma a with no muscle invasion seen on biopsy specimen. Penile growth biopsy showed a moderately differentiated squamous cell carcinoma. FNAC (Fine needle aspiration cytology) of in guinal lymph nodes came out as non-specific reactive lymphadenitis.

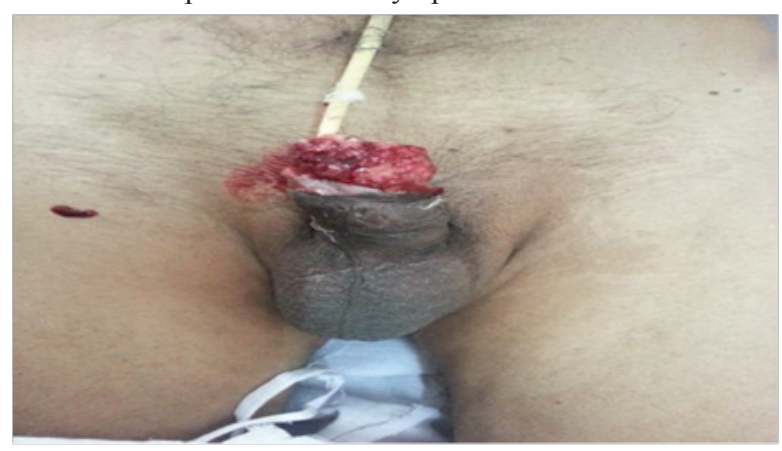

Figure I Penile growth at presentation.

The patient was then planned for a partial penectomy along with transurethral resection of the bladder tumour. The patient underwent a partial penectomy Figure 3 along with transurethral resection of the bladder tumour. The pathology results from the TURBT (Transurethral resection of bladder tumour) revealed a low grade transitional cell carcinoma, with no muscle invasion (Figures $4 \&$ Figure 5). Penile resection specimen showed a moderately differentiated squamous cell carcinoma with no lympho vascular invasion with the re-sected 
margin being negative for tumour (Figures $6 \&$ Figure 7). The TNM stage of the penile and bladder tumour was T1N0M0 and T1NOM0, respectively. The final diagnosis was double primary cancer of the penis and bladder. The follow-up check Cystoscopy and CT scan of the abdomen and pelvis done six months after the operation showed no evidence of recurrence. The inguinal lymphadenopathy too subsided post-operative lyon antibiotics treatment, presumably being reactive in origin. Patient reports no lower urinary tract symptoms and has good stream and minimal post void volume. ${ }^{8}$

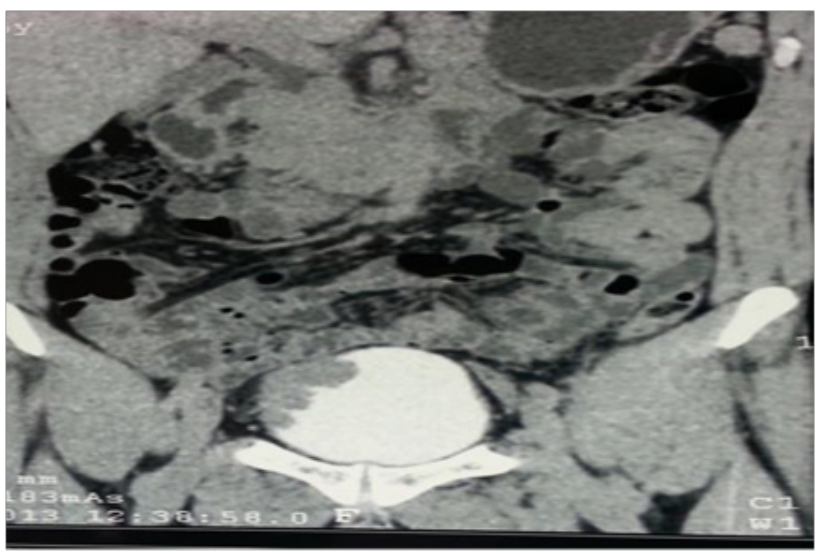

Figure 2 CECT showingbladder mass on posterolateral wall.

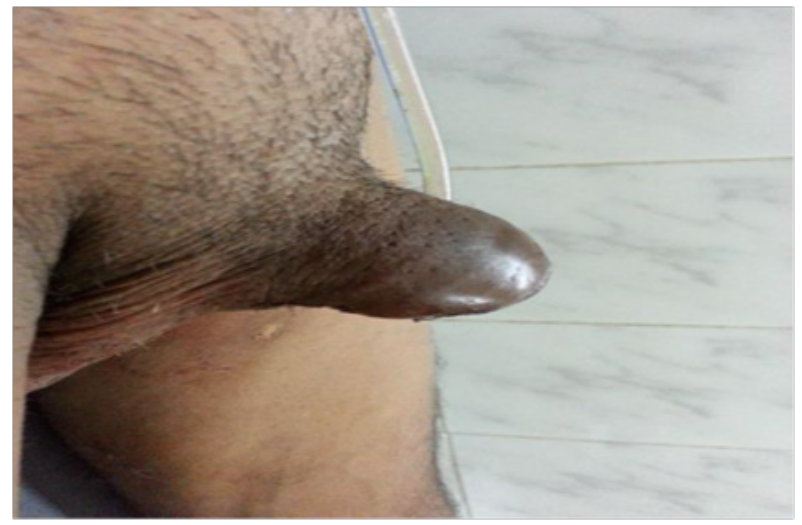

Figure $3 \mathrm{Pt}$ after partial penectomy.

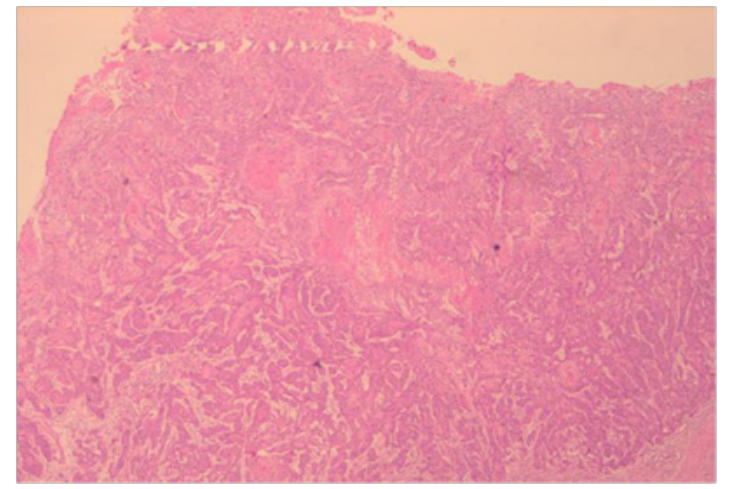

Figure 4sa Low power magnification of penile growth.

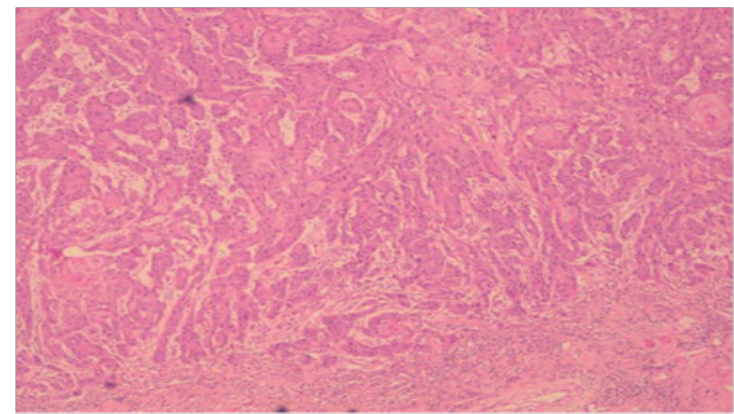

Figure 5 High power magnification of penile growth.

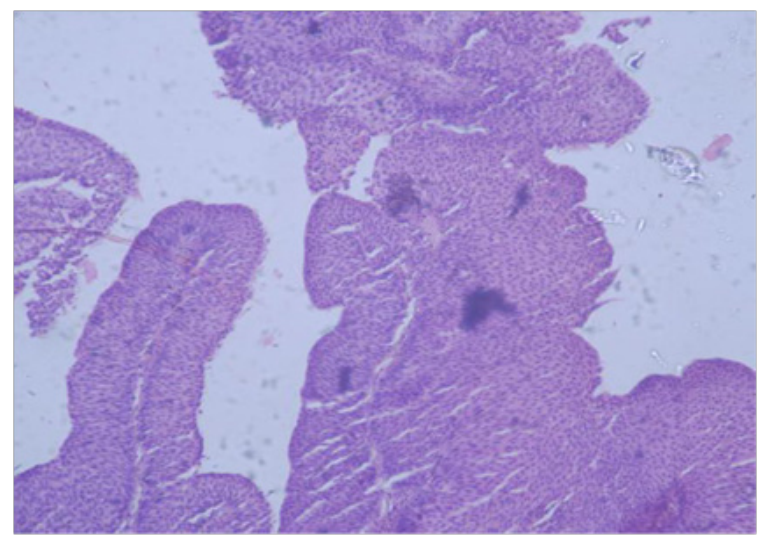

Figure 6 Low power magnification of bladder tumor.

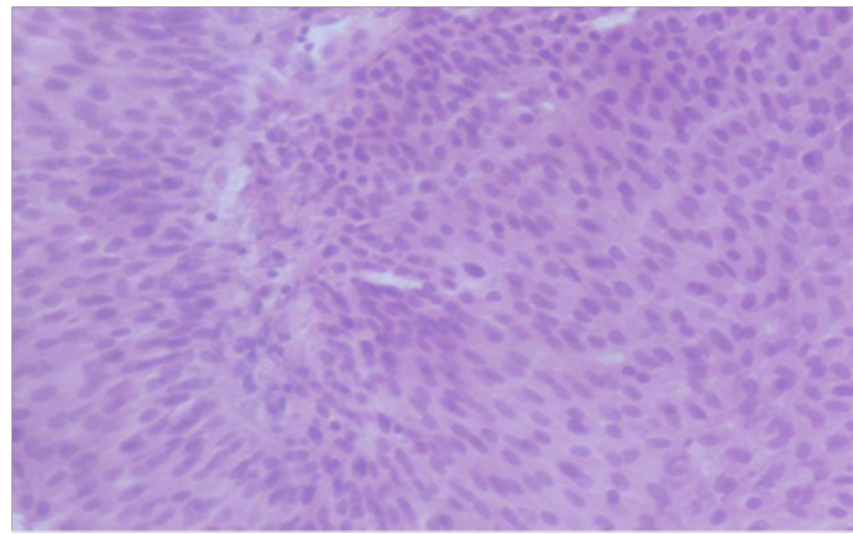

Figure 7 High power magnification of bladder.

\section{Discussion}

Although Warren and Gates criteria are useful, Moertel et al. ${ }^{9}$ has suggested a classification including multiple primary cancers and multicentric cancers to avoid confusion between the two.

1. Group I-multiple primary cancers occurring in organs with the same histology.

2. Group II-multiple primary cancers from different tissues.

Group I is further subdivided into: 
a. Group A-cancers that occur in the same tissue and organ

b. Group B-cancers that are from the same tissue and different organs Group C-cancers that occur in bilateral organs (i.e., breast, ovary, etc.).

Group III-cancers from different tissues and organs that concurrently exist with group I, forming multiple primary cancers of three or more cancers. The reason behind development of MPC has been a source of much debate with reasons ranging from family history, immunologic and genetic defects (for example, Li-Fraumeni or Beckwith-Wiedemann syndrome) to prolonged exposure to carcinogens, radiation and chemotherapy for the primary cancer and the field cancerization theory suggested by Slaughter et al. in $1953 .{ }^{10}$ The concept being this theory is that when the body is exposed to carcinogens, other organs besides the organ with cancer are also exposed to the carcinogen and carry a high risk of cancer.

Penile cancer is thought to be the result of infection from human papilloma virus, phimosis, poor hygiene and cigarette smoking, ${ }^{11}$ while bladder cancer is due to schistosomiasis, chemical exposure, pelvic radiotherapy and smoking. ${ }^{12}$ The history of our patient, along with the above suggests cigarette smoking as the possible etiology. Also to be considered as a possible etiological factor in our case is his exposure to pesticides, ultraviolet radiation exposure and poor hygiene. Prior exposure to insecticides, herbicides, and fungicides or seed treatments are associated with an increased incidence of SCC. ${ }^{13}$ Therapy regarding MPC is not standardized but patient characteristics and tumor biology are the decision drivers. In general surgery with curative intent is advised if possibility of a R0 resection is there. Organ preservation is of utmost importance in penile cancer so as to provide a better quality of life to the patient along with minimal morbidity, especially when a favorable histology (stages Tis, Ta, T1; grades 1 and 2 ) is present as they are at a low risk for local progression and distant metastatic spread..$^{14}$ In our case partial penectomy and TURBT proved to be sufficient. ${ }^{15}$

Increasing age, improved survival rates, better diagnostic techniques, changing lifestyle are suggested as the possible etiology for the rising rate of MPC. ${ }^{3}$ Causal mechanisms of multiple primary cancers include genes, the environment, treatment effects, and combinations of these three mechanisms. When researchers focus on genes as causal mechanisms, they often concentrate on cases diagnosed earlier in life (i.e., younger than age 55). Data indicate that when a person is genetically predisposed to develop cancer, he or she more often will do so earlier in life than a person who develops cancer sporadically. A study linking populations from the Utah Cancer Registry, the Genealogical Society of Utah (a database of 1 million Utah descendants), and Utah death certificates found that first-degree relatives of probands were more likely to develop various types of cancers at a younger age than controls. A significant association with the development of cancers also was linked to smoking and alcohol consumption behaviours.

\section{Acknowledgements}

None.

\section{Conflict of interest}

The authors declare that there is no conflict of interests regarding the publication of this manuscript.

\section{References}

1. Billroth T. General surgical pathology and therapeutics in 51 Vorlesungen: a text book for students and physicians in fifty-one lectures. Berlin DE, et al. editors. 14th ed. 1889

2. Warren S, Gates O. Multiple primary malignant tumors; Surgery of literature and statistical study. Am J Cancer. 1932;16:1358-1414.

3. Koutsopoulos AV, Dambaki KI, Datseris G, et al. A novel combination of multiple primary carcinomas: Urinary bladder transitional cell carcinoma, prostate adenocarcinoma and small cell lung carcinoma-report of a case and review of the literature. World J Surg Oncol. 2005;3:51

4. Wegner HE. Multiple primary cancers in urologic patients: Audit of 19-year experience in Berlin and review of the literature. Urology. 1992;39(3):231-236.

5. Ray P, Sharifi R, Ortolano V, et al. Involvement of the genito urinary system in multiple primary malignant neoplasm: a review. J Clin Oncol. 1983;1(9):574-581.

6. Parkin DM. The global burden of urinary bladder cancer. Scand J Urol Nephrol. 2008;Suppl(218):12-20.

7. Barnholtz-Sloan JS, Maldonado JL, Pow-sang J, et al. Incidence trends in primary malignant penile cancer. Urol Onco. 2007;25(5):361-367.

8. Yong Soo Cho, Jung-Ae Lee, Si Bum Kim, et al. A case of synchronous double primary cancer of the penis and urinary bladder. Cancer Res Treat. 2000;42(1):53-56.

9. Moertel CG. Multiple primary malignant neoplasms: Historical perspectives. Cancer. 1977;40(4):1786-1792.

10. Slaughter DP, Southwick HW, Smejka 1W. Field cancerization in oral stratified squamous epithelium; Clinical implications of multicentric origin. Cancer. 1953;6(5):963-968.

11. Dillner J, Von Krogh G, Horenblas S, et al. Etiology of squamous cell carcinoma of the penis. Scand J Urol Nephrol. 2000;205:189-193.

12. Weinberg DM, Ross RK, Mack TM, et al. Bladder cancer etiology. A different perspective. Cancer. 1983;51(4):675-680.

13. Gallagher RP, Bajdik CD, Fincham S. Chemical exposures, medical history, and risk of squamous and basal cell carcinoma of the skin. Cancer Epidemiol Biomarkers Prev. 1996;5(6):419-424.

14. Martins FE, Rodrigues RN, Lopes TM. Organ-preserving surgery for penile carcinoma. Adv Urol. 2008;2008:634216.

15. Goldgar DE, Easton DF, Cannon-Albright LA, et al. Systematic population-based assessment of cancer risk in first-degree relatives of cancer probands. J Natl Cancer Inst. 1994;86(21):1600-1608. 